\title{
BEURLING'S THEOREM FOR RIEMANNIAN SYMMETRIC SPACES II
}

\author{
RUDRA P. SARKAR AND JYOTI SENGUPTA \\ (Communicated by Michael T. Lacey)
}

\begin{abstract}
We prove two versions of Beurling's theorem for Riemannian symmetric spaces of arbitrary rank. One of them uses the group Fourier transform and the other uses the Helgason Fourier transform. This is the master theorem in the quantitative uncertainty principle.
\end{abstract}

\section{INTRODUCTION}

The uncertainty principle in harmonic analysis on $\mathbb{R}^{n}$ is the paradigm which says that a function and its Fourier transform cannot both decay very rapidly at infinity. This principle has several quantitative versions, which was proved by Hardy, Morgan, Gelfand and Shilov, Cowling and Price, etc. (see [6], [8] [16] and the references therein). The following theorem by Hörmander in the case of $\mathbb{R}([11])$ is the strongest theorem in this genre in the sense that it implies the theorems of Hardy, Morgan, Gelfand and Shilov, and Cowling and Price.

Theorem 1.1 (Hörmander 1991). Let $f \in L^{1}(\mathbb{R})$. Then

$$
\int_{\mathbb{R}} \int_{\mathbb{R}}|f(x)||\widehat{f}(y)| e^{|x y|} d x d y<\infty
$$

implies $f=0$ almost everywhere.

Hörmander attributes this theorem to A. Beurling. We will follow his practice and call Theorem 1.1 Beurling's theorem throughout this paper. The aim of this article is to prove an analogue of Beurling's theorem for Riemannian symmetric spaces $X$ of the noncompact type. We recall that such a space is of the form $G / K$ where $G$ is a noncompact connected semisimple Lie group with finite centre and $K \subset G$ is a maximal compact subgroup. We prove two different versions of the theorem. In the first version we consider functions on $X$ as right $K$-invariant functions on $G$ and we use the operator valued group Fourier transform to formulate the theorem, while in the second version we use the Helgason-Fourier transform, that is, the geometric Fourier transform on $X$. From different points of view different versions appear to be the correct analogue of Beurling's theorem for $\mathbb{R}$. The precise statements of the theorems and their proofs appear in Sections 3 and 4 of our article. In Section 5 we have indicated why Beurling's theorem should be regarded as

Received by the editors November 9, 2006.

2000 Mathematics Subject Classification. Primary 22E30, 43A85.

Key words and phrases. Beurling's theorem, uncertainty principle, symmetric space, Radon transform. 
the master theorem in the quantitative uncertainty principle. This paper is a sequel to [13], where we established Beurling's theorem for rank 1 symmetric spaces.

\section{Notation AND PRELIMINARIES}

In this section we introduce the notation that we employ and state some basic pertinent facts regarding harmonic analysis on $X$. We also prove a lemma that we use subsequently. The pair $(G, K)$ is as described in the introduction. We let $G=K A N$ denote a fixed Iwasawa decomposition of $G$. Let $\mathfrak{g}, \mathfrak{k}, \mathfrak{a}$, and $\mathfrak{n}$ denote the Lie algebras of $G, K, A$, and $N$, respectively. We choose and keep fixed throughout a system of positive restricted roots for the pair $(\mathfrak{g}, \mathfrak{a})$, which we denote by $\Sigma^{+}$. The subset of positive indivisible roots will be denoted by $\Sigma_{0}^{+}$. The multiplicity of a root $\alpha \in \Sigma^{+}$will be denoted by $m_{\alpha}$. As usual the half-sum of the elements of $\Sigma^{+}$counted with their multiplicities will be denoted by $\rho$. Let $H: G \longrightarrow \mathfrak{a}$ be the Iwasawa projection associated to the Iwasawa decomposition, $G=K A N$. Then $H$ is left $K$-invariant and right $M N$-invariant where $M$ is the centralizer of $A$ in $K$. The Weyl group of the pair $(G, A)$ will be denoted by $W$. Let $\mathfrak{a}^{*}$ be the dual of $\mathfrak{a}$. For $\lambda \in \mathfrak{a}^{*}$ (respectively $H \in \mathfrak{a}$ ) we denote by $\lambda^{+}$(respectively $H^{+}$) the unique Weyl translate of $\lambda$ (respectively $H$ ) that belongs to the closure of the positive Weyl chamber $\mathfrak{a}_{+}^{*}$ (respectively $\mathfrak{a}_{+}$). Let $A^{+}=\exp \mathfrak{a}_{+}$.

We have the a-valued function $A(x, k)$ on $X \times K$ defined by

$$
A(g K, k)=-H\left(g^{-1} k\right), \quad g \in G, k \in K .
$$

Note that $A$ descends to a function, also denoted by $A: X \times K / M \longrightarrow \mathfrak{a}$, since $H$ is right $M$-invariant.

We recall that the Killing form $B$ restricted to $\mathfrak{a}$ is a positive definite inner product on $\mathfrak{a}$ and it gives a $W$-equivariant isomorphism of $\mathfrak{a}$ with $\mathfrak{a}^{*}$. For $\lambda \in \mathfrak{a}^{*}$ we denote the corresponding element in $\mathfrak{a}$ by $H_{\lambda}$. Let $n$ be the rank of $X$. We will identify $\mathfrak{a}$ and $\mathfrak{a}^{*}$ with $\mathbb{R}^{n}$, as an inner product space, with the inner product on $\mathbb{R}^{n}$ being the pull-back of the Killing form. This inner product on $\mathbb{R}^{n}$ as well as on $\mathfrak{a}, \mathfrak{a}^{*}$ will be referred to as the Killing inner product and will be denoted by $\langle$,$\rangle .$ The associated norm will be denoted by $|\cdot|$. We hope that this symbol will not be confused with the absolute value symbol. Since $\exp : \mathfrak{a} \longrightarrow A$ is an isomorphism, as a group $A$ can be identified with $\mathbb{R}^{n}$.

For $x \in G$, we define $\sigma(x)=d(x K, K)$ where $d$ is the canonical distance function for $X=G / K$ coming from the Riemann metric on $X$ induced by the Killing form restricted to $\mathfrak{p}$. Here $\mathfrak{g}=\mathfrak{k} \oplus \mathfrak{p}$ (Cartan decomposition) and $\mathfrak{p}$ can be identified with the tangent space at $e K$ of $G / K$. The function $\sigma(x)$ is $K$-biinvariant and continuous. Note that for $x=k_{1} a k_{2}, k_{1}, k_{2} \in K, a \in \overline{A^{+}}, \sigma(x)=\sigma(a)=|\log a|$, the Killing norm of $\log a$, where $\log a$ is the unique element in $\mathfrak{a}$ such that $\exp (\log a)=a$.

On $X$ we fix the measure $d x$ which is induced by the metric we obtain from $B$. As the metric is $G$-invariant, so is $d x$. On $G$ we fix the Haar measure $d g$ satisfying

$$
\int_{X} f(x) d x=\int_{g} f(g) d g
$$

for every integrable function $f$ on $X$ which we also consider as a right $K$-invariant function on $G$. While dealing with functions on $X$, we may slur over the difference between the two measures.

Through the identification of $A$ with $\mathbb{R}^{n}$ we use the Lebesgue measure on $\mathbb{R}^{n}$ as the Haar measure $d a$ on $A$. As usual on the compact group $K$ we fix the normalized 
Haar measure $d k$ (i.e. $\operatorname{vol}(K)=\int_{K} d k=1$ ). Finally we fix the Haar measure $d n$ on $N$ by the condition that

$$
\int_{G} f(g) d g=\int_{A} \int_{N} \int_{K} f(a n k) d k d n d a
$$

holds for every integrable function $f$ on $G$.

Definition 2.1. For a function $f$ in $C_{c}^{\infty}(X)$, the Helgason Fourier transform $\tilde{f}$ of $f$ is defined by

$$
\widetilde{f}(\lambda, k)=\int_{X} e^{(-i \lambda+\rho)(A(x, k))} f(x) d x ; \lambda \in \mathfrak{a}^{*}, k \in K .
$$

Note that $\tilde{f}$ descends to a function on $\mathfrak{a}^{*} \times K / M$. For $f \in L^{1}(X)$, there exists a subset $K^{\prime} \subset K$ of full Haar measure such that $\widetilde{f}(\lambda, k)$ exists for all $k \in K^{\prime}$ and $\lambda \in T_{\rho}$ where $T_{\rho}$ is the tube $\mathfrak{a}^{*}+i C_{\rho} \subset \mathfrak{a}_{\mathbb{C}}^{*}$ (the complexification of $\mathfrak{a}^{*}$ ), $C_{\rho}$ being the convex hull of the Weyl orbit of $\rho$. In fact for every fixed $k \in K^{\prime}$, the function $\lambda \mapsto \widetilde{f}(\lambda, k)$ is continuous in $T_{\rho}$ and holomorphic in its interior (see [9]).

Definition 2.2. For $f$ in $C_{c}^{\infty}(X)$, the Radon transform $\mathcal{R} f$ of $f$ is defined by

$$
\mathcal{R} f(k, a)=e^{\rho(\log a)} \int_{N} f(k a n) d n ; k \in K, a \in A .
$$

The Radon transform $\mathcal{R} f$ descends to a function on $K / M \times A$ and (as in the case of $\widetilde{f}$ ) we continue to denote this function by $\mathcal{R} f$. It is well known that for $f \in L^{1}(X), \mathcal{R} f$ is well defined and in fact $\mathcal{R} f \in L^{1}(K \times A ; d k d a)([4])$.

Let $\widehat{K}_{M}$ denote the set of equivalence classes of irreducible unitary representations of $K$ which contain an $M$-fixed vector. For $\delta \in \widehat{K}_{M}$, let $V_{\delta}$ be its carrier space and let $V_{\delta}^{M}$ be the subspace of $V_{\delta}$ of the $M$-fixed vectors. Let $\operatorname{dim} V_{\delta}=d(\delta)$ and $\operatorname{dim} V_{\delta}^{M}=l$. Let $v_{1}, v_{2}, \ldots, v_{d(\delta)}$ be a fixed orthonormal basis of $V_{\delta}$ whose first $l$ elements form a basis of $V_{\delta}^{M}$. For a suitable complex valued function $f$ on $X=G / K$ and for $\delta \in \widehat{K}_{M}$, we define its $\delta$-th component $f^{\delta}$ as ([10], p. 276)

$$
f^{\delta}(x)=d(\delta) \int_{K} f(k x) \delta\left(k^{-1}\right) d k, x \in G .
$$

Then $f^{\delta}$ is a $\operatorname{Hom}\left(V_{\delta}, V_{\delta}\right)$ valued function on $X$ and $f^{\delta}(k x)=\delta(k) f^{\delta}(x)$.

The generalized spherical function of class $\delta$ is defined as ([10], p. 233)

$$
\Phi_{\lambda, \delta}(x)=\int_{K} e^{-(i \lambda+\rho)\left(H\left(x^{-1} k\right)\right)} \delta(k) d k,
$$

for $\lambda \in \mathfrak{a}_{\mathbb{C}}^{*}$ and $x \in G$. Then $\Phi_{\lambda, \delta}(k x)=\delta(k) \Phi_{\lambda, \delta}(x)$ for $k \in K, x \in G$. When $\delta$ is the trivial representation of $K$, then $\Phi_{\lambda, \delta}$ is the elementary spherical function $\phi_{\lambda}$ with parameter $\lambda$, i.e.

$$
\phi_{\lambda}(x)=\int_{K} e^{-(i \lambda+\rho)\left(H\left(x^{-1} k\right)\right)} d k .
$$

Clearly, the adjoint of $\Phi_{\lambda, \delta}(x)$ is

$$
\Phi_{\lambda, \delta}(x)^{*}=\int_{K} e^{(i \bar{\lambda}-\rho) H\left(x^{-1} k\right)} \delta\left(k^{-1}\right) d k
$$


and $\Phi_{\lambda, \delta}(k x)^{*}=\Phi_{\lambda, \delta}(x)^{*} \delta\left(k^{-1}\right)$. Both $\Phi_{\lambda, \delta}(x)$ and $\Phi_{\lambda, \delta}(x)^{*}$ are right $K$-invariant functions when regarded as functions on $G$. Note also that as $H$ is right $M$ invariant, $\Phi_{\lambda, \delta}(x)^{*}$ is a $\operatorname{Hom}\left(V_{\delta}, V_{\delta}^{M}\right)$ valued function. Also,

$$
\left\|\Phi_{\lambda, \delta}(x)^{*}\right\|_{2} \leq \int_{K}\left|e^{(i \bar{\lambda}-\rho) H\left(x^{-1} k\right)}\right|\left\|\delta\left(k^{-1}\right)\right\|_{2} d k=\sqrt{d(\delta)} \phi_{i \lambda_{I}}(x) .
$$

Here $\|\cdot\|_{2}$ denotes the Hilbert-Schmidt norm and $\lambda_{I}$ denotes the imaginary part of $\lambda$. Now using the estimate for $\phi_{\lambda}$ (see [7], p. 158), we have for $a \in A$,

$$
\left\|\Phi_{\lambda, \delta}(a)^{*}\right\|_{2} \leq \sqrt{d(\delta)} e^{-\left(\lambda_{I}\right)^{+}\left(\log a^{+}\right)} \Xi(a) .
$$

We also need the following upper and lower estimates for $\Xi(x)=\phi_{0}(x)$ (see [7], Theorem 4.6.4 and Theorem 4.6.5): There exists a constant $C>0$ such that for all $a \in \overline{A^{+}}\left(A^{+}=\exp \mathfrak{a}^{+}\right)$,

$$
e^{-\rho(\log a)} \leq \Xi(a) \leq C e^{-\rho(\log a)}(1+|\log (a)|)^{d}
$$

where $d$ is the number of short positive roots.

We define

$$
\widehat{f^{\delta}}(\lambda)=\int_{G} \Phi_{\bar{\lambda}, \delta}(x)^{*} f^{\delta}(x) d x
$$

Using the Cartan decomposition $G=K \overline{A^{+}} K$, we have

$$
\widehat{f^{\delta}}(\lambda)=\int_{\overline{A^{+}}} \Phi_{\bar{\lambda}, \delta}(a)^{*} f^{\delta}(a) J(a) d a
$$

where $J(a)$ is the Jacobian of the Cartan decomposition. Then $\widehat{f^{\delta}}(\lambda)$ is also a $\operatorname{Hom}\left(V_{\delta}, V_{\delta}^{M}\right)$ valued function.

Also,

$$
\begin{aligned}
\widehat{f^{\delta}}(\lambda) & =\int_{G} \Phi_{\bar{\lambda}, \delta}(x)^{*} f^{\delta}(x) d x \\
& =\int_{G} \Phi_{\bar{\lambda}, \delta}(x)^{*} \int_{K} f(k x) \delta\left(k^{-1}\right) d k d x \\
& =\int_{K} \int_{G} \Phi_{\bar{\lambda}, \delta}(x)^{*} f(k x) d x \delta\left(k^{-1}\right) d k .
\end{aligned}
$$

Substituting $k x=y$, we get

$$
\begin{aligned}
\widehat{f^{\delta}}(\lambda) & =\int_{K} \int_{G} \Phi_{\bar{\lambda}, \delta}(y)^{*} \delta(k) f(y) d y \delta\left(k^{-1}\right) d k \\
& =\int_{G} \Phi_{\bar{\lambda}, \delta}(y)^{*} f(y) d y .
\end{aligned}
$$

Definition 2.3. For $f \in L^{1}(X)$, the Abel transform of $f^{\delta}, \mathcal{A} f^{\delta}$ is defined by

$$
\mathcal{A} f^{\delta}(a)=e^{\rho(\log a)} \int_{N} f^{\delta}(a n) d n, a \in A
$$

Since $\delta(m) f^{\delta}($ an $)=f^{\delta}(\operatorname{man})=f^{\delta}($ amn $)=f^{\delta}\left(a m n m^{-1} m\right)=f^{\delta}\left(a m n_{1}\right)$ where $m n m^{-1}=n_{1} \in N$ and the Jacobian for the substitution $m n m^{-1}=n_{1}$ is 1 in the integration on $N$, it is clear that $\mathcal{A} f^{\delta}$ is a $\operatorname{Hom}\left(V_{\delta}, V_{\delta}^{M}\right)$ valued function on $A$. 
Note that

$$
\begin{aligned}
\int_{K} \mathcal{R} f(k, a) \delta\left(k^{-1}\right) d k & =e^{\rho(\log a)} \int_{N} \int_{K} f(k a n) \delta\left(k^{-1}\right) d k d n \\
& =e^{\rho(\log a)} \int_{N} f^{\delta}(\text { an }) d n \\
& =\mathcal{A} f^{\delta}(a)
\end{aligned}
$$

Therefore

$$
\left\|\mathcal{A} f^{\delta}(a)\right\|_{2} \leq \int_{K}|\mathcal{R} f(k, a)|\left\|\delta\left(k^{-1}\right)\right\|_{2} d k=\sqrt{d(\delta)} \int_{K}|\mathcal{R} f(k, a)| d k .
$$

Hence we obtain

$$
\int_{A}\left\|\mathcal{A} f^{\delta}(a)\right\|_{2} d a \leq \sqrt{d(\delta)} \int_{A} \int_{K}|\mathcal{R} f(k, a)| d k d a<\infty
$$

since $\mathcal{R} f \in L^{1}(K \times A ; d k d a)$ as mentioned above. Therefore in particular every matrix entry of $\mathcal{A} f^{\delta}$ with respect to our chosen basis $v_{1}, v_{2}, \ldots, v_{d(\delta)}$ is a complex valued $L^{1}$-function on $A$.

Before proving Lemma 2.4, we make some remarks concerning the Euclidean Fourier and Radon transforms. If $(V,\langle\rangle$,$) is a finite dimensional inner product$ space over $\mathbb{R}$, then for $f \in L^{1}(V)$ we define its Euclidean Fourier transform $\tilde{f}$ by

$$
\tilde{f}(\lambda)=\int_{V} f(x) e^{-i \lambda(x)} d x ; \lambda \in V^{*} .
$$

Identifying $V^{*}$ with $V$ by means of the inner product, we can write $\tilde{f}$ as $\tilde{f}(\lambda)=$ $\int_{V} f(x) e^{-i\langle\lambda, x\rangle} d x ; \lambda \in V$.

Let $S^{n-1}$ denote the unit sphere in $\mathbb{R}^{n}$. For a suitable function $f$ on $\mathbb{R}^{n}$, the Euclidean Radon transform $\mathcal{R}^{E} f$ is a function on $S^{n-1} \times \mathbb{R}$, defined by

$$
\mathcal{R}^{E} f(\omega, r)=\mathcal{R}_{\omega}^{E} f(r)=\int_{x \cdot \omega=r} f(x) d \sigma_{x},
$$

where $d \sigma_{x}$ denotes the $(n-1)$-dimensional Lebesgue measure on the hyperplane $x \cdot \omega=r$ and $x \cdot \omega$ is the canonical inner product of $x$ and $\omega$, i.e., $x \cdot \omega=\sum_{i=1}^{n} x_{i} \omega_{i}$. We will use both the symbols $\mathcal{R}^{E} f(\omega, r)$ and $\mathcal{R}_{\omega}^{E} f(r)$ to denote the same object. Note that when $f \in L^{1}\left(\mathbb{R}^{n}\right)$, for any fixed $\omega \in S^{n-1}, \mathcal{R}^{E} f(\omega, r)$ exists for almost every $r \in \mathbb{R}$ and is an $L^{1}$-function on $\mathbb{R}$. It is also well known that (see [5], p. 185)

$$
\widetilde{\mathcal{R}_{\omega}^{E} f}(\lambda)=\widetilde{f}(\lambda \omega) \text {. }
$$

Here $\widetilde{\mathcal{R}_{\omega}^{E} f}(\lambda)=\int_{\mathbb{R}} \mathcal{R}_{\omega}^{E} f(r) e^{-i \lambda r} d r$ and $\widetilde{f}(\lambda \omega)=\int_{\mathbb{R}^{n}} f(x) e^{-i x \cdot \lambda \omega} d x$. It is easy to verify that the above definition of the Radon transform is meaningful if we replace the canonical inner product on $\mathbb{R}^{n}$ by an arbitrary (positive definite) inner product. Furthermore equation (2.5) remains valid in this setup when $\tilde{f}$ is defined by means of this inner product.

Let us also point out here that Theorem 1.1 is true if we replace the canonical inner product by any other inner product on $\mathbb{R}$ provided we define the Fourier transform using this other inner product.

We will use the following lemma to prove our main result.

Lemma 2.4. Let $f$ be a function in $L^{1}(X)$. Let $\mathcal{A}\left(f^{\delta}\right)^{\sim}(\lambda)$ be the matrix whose entries are the Euclidean Fourier transform of the entries of the matrix $\mathcal{A} f^{\delta}$ with respect to the basis $v_{1}, v_{2}, \ldots, v_{d(\delta)}$ of $V_{\delta}$. Then $\mathcal{A}\left(f^{\delta}\right)^{\sim}(\lambda)=\widehat{f^{\delta}}(\lambda)$ for all $\lambda \in \mathfrak{a}^{*}$. 
In fact this relation remains valid for $\lambda \in \mathfrak{a}_{\mathbb{C}}^{*}$ for which the left and the right sides of the equality exist.

Proof. From the definition using Fubini's theorem, we get

$$
\widehat{f^{\delta}}(\lambda)=\int_{G} \Phi_{\bar{\lambda}, \delta}(x)^{*} f^{\delta}(x) d x=\int_{K} \int_{G} e^{(i \lambda-\rho) H\left(x^{-1} k\right)} \delta\left(k^{-1}\right) f^{\delta}(x) d x d k .
$$

Substituting $x=k y$ in the above integral, we get $\widehat{f^{\delta}}(\lambda)=\int_{G} e^{(i \lambda-\rho) H\left(y^{-1}\right)} f^{\delta}(y) d y$. We use the Iwasawa decomposition $G=A N K$ which has Jacobian 1, i.e., $d g=$ $d a d n d k$ and we write $y=a n k$ according to this decomposition to obtain

$$
\begin{aligned}
\widehat{f^{\delta}}(\lambda) & =\int_{A \times N \times K} e^{(i \lambda-\rho) H\left(k^{-1} n^{-1} a^{-1}\right)} f^{\delta}(\text { an }) d a d n d k \\
& =\int_{A \times N} e^{(i \lambda-\rho)(-\log a)} f^{\delta}(\text { an }) d a d n \\
& =\int_{A}\left(e^{\rho(\log a)} \int_{N} f^{\delta}(\text { an }) d n\right) e^{-i \lambda(\log a)} d a \\
& =\mathcal{A}\left(f^{\delta}\right)^{\sim}(\lambda) .
\end{aligned}
$$

In the second step we have used that $H$ is left $K$-invariant, right $N$-invariant, and $A$ normalizes $N$. Hence $H\left(k^{-1} n^{-1} a^{-1}\right)=-\log a$.

We will conclude this section by stating the following uniform asymptotic estimate of the spherical Plancherel density (see [1]):

$$
\mu(\lambda)=|c(\lambda)|^{-2} \asymp \Pi_{\alpha \in \sum_{0}^{+}}\langle\lambda, \alpha\rangle^{2}(1+|\langle\lambda, \alpha\rangle|)^{m_{\alpha}+m_{2 \alpha}-2}, \text { for } \lambda \in \mathfrak{a}^{*} .
$$

Here $f \asymp g$ means $c_{1} g(\lambda) \leq f(\lambda) \leq c_{2} g(\lambda)$ for $\lambda \in \mathfrak{a}^{*},|\lambda|$ large, $c_{1}, c_{2}$ being positive constants.

We are now in a position to state our main results. In the proofs of the theorems we will use Fubini's theorem freely without explicitly mentioning it every time.

\section{FIRST VERSION}

This version uses the group Fourier transform.

Theorem 3.1. Let $f \in L^{2}(X)$. If

$$
\int_{X} \int_{\mathfrak{a}^{*}}|f(x)|\|\widehat{f}(\lambda)\|_{2} e^{\sigma(x)|\lambda|} \Xi(x) d x \mu(\lambda) d \lambda<\infty
$$

where $|\cdot|$ is the Killing-norm on $\mathfrak{a}^{*}$, then $f=0$ almost everywhere.

We will first prove a proposition.

Proposition 3.2. Let $f$ be as in Theorem 3.1. Then $f \in L^{1}(X)$.

Proof. Since $f \in L^{2}(X), f$ is a locally integrable function on $X$. Condition (3.1) implies that

$$
\int_{X}|f(x)| e^{\sigma(x)|\lambda|} \Xi(x) d x<\infty
$$

for almost every $\lambda \in \mathfrak{a}^{*}$ where $\widehat{f}(\lambda) \neq 0$.

Let $S_{f}=\left\{\lambda \in \mathfrak{a}^{*}: \widehat{f}(\lambda) \neq 0\right\}$. Then $S_{f}$ is well defined up to a set of Plancherel measure zero (E, say), since $f \in L^{2}(X)$. The Plancherel density $\mu(\lambda)$ is real analytic. Hence $E$ has Lebesgue measure zero. 
Now there are two possible situations:

(a) $S_{f}$ has infinite Lebesgue measure,

(b) $S_{f}$ has finite Lebesgue measure.

Case (a). Using the Cartan decomposition $G=K \overline{A^{+}} K$, we obtain

$$
\int_{K} \int_{\overline{A^{+}}}|f(k a)| e^{\sigma(a)|\lambda|} \Xi(a) J(a) d a d k=\int_{\overline{A^{+}}}|f|_{0}(a) e^{\sigma(a)|\lambda|} \Xi(a) J(a) d a<\infty,
$$

for $\lambda \in S_{f}$. Here $|f|_{0}$ is the biinvariant component of $|f|$. We use the (lower) estimate in (2.3) of $\Xi(a)$ for $a \in \overline{A^{+}}$to obtain

$$
\int_{\overline{A^{+}}}|f|_{0}(a) e^{\sigma(a)|\lambda|} e^{-\rho(\log a)} J(a) d a<\infty .
$$

Our aim is to show that $\int_{X}|f(x)| d x<\infty$, which is the same as showing

$$
\int_{K \times \overline{A^{+}}}|f(k a)| J(a) d a d k=\int_{\overline{A^{+}}}|f|_{0}(a) J(a) d a<\infty .
$$

As $S_{f}$ has infinite measure, we can get a $\lambda_{0} \in S_{f}$ with $\left|\lambda_{0}\right|>(m+1)|\rho|$ for any fixed $m>1$. Therefore

$$
\sigma(a)\left|\lambda_{0}\right|>\sigma(a)(m+1)|\rho| \geq|(m+1) \rho(\log a)|=(m+1) \rho(\log a) \text { for } a \in \overline{A^{+}} .
$$

Applying this in (3.2), we see that

$$
\int_{\overline{A^{+}}}|f|_{0}(a) e^{m \rho(\log a)} J(a) d a<\infty
$$

and hence

$$
\int_{\overline{A^{+}}}|f|_{0}(a) J(a) d a<\infty
$$

since for $a \in \overline{A^{+}}, \rho(\log a) \geq 0$.

Case (b). As $\widehat{f} \not \equiv 0$, there exists $\lambda_{0} \neq 0, \lambda_{0} \in S_{f}$. Let $\left|\lambda_{0}\right|=r>0$. Then from (3.1) we have $\int_{X}|f(x)| e^{r \sigma(x)} \Xi(x) d x<\infty$. Now using the Cartan decomposition as in case (a) we get,

$$
\int_{\overline{A^{+}}}|f|_{0}(a) e^{r \sigma(a)} \Xi(a) J(a) d a<\infty
$$

Recall that

$$
\widehat{f^{\delta}}(\lambda)=\int_{G} \Phi_{\bar{\lambda}, \delta}^{*}(x) f(x) d x .
$$

Hence for $1 \leq i \leq d(\delta)$ and $1 \leq j \leq l$,

$$
\widehat{f^{\delta}}(\lambda)_{i j}=\int_{G}\left(\Phi_{\bar{\lambda}, \delta}^{*}\right)_{i j}(x) f(x) d x
$$

where $\widehat{f^{\delta}}(\lambda)_{i j}$ and $\left(\Phi_{\bar{\lambda}, \delta}^{*}\right)_{i j}$ are the $(i, j)$-th matrix entries of $\widehat{f^{\delta}}(\lambda)$ and $\Phi_{\bar{\lambda}, \delta}^{*}$, respectively. Hence, using (2.1),

$$
\begin{aligned}
\left|\widehat{f^{\delta}}(\lambda)_{i j}\right| & \leq \int_{G}\left|\left(\Phi_{\bar{\lambda}, \delta}^{*}\right)_{i j}(x)\right||f(x)| d x \\
& \leq \sqrt{d(\delta)} \int_{G}|f(x)| \phi_{-i \lambda_{I}}(x) d x .
\end{aligned}
$$


Using Cartan decomposition, $G=K \overline{A^{+}} K$, and (2.2), we get from the above that

$$
\begin{aligned}
\left|\widehat{f^{\delta}}(\lambda)_{i j}\right| & \leq \sqrt{d(\delta)} \int_{\overline{A^{+}}}|f|_{0}(a) \phi_{-i \lambda_{I}}(a) J(a) d a \\
& \leq \sqrt{d(\delta)} \int_{\overline{A^{+}}}|f|_{0}(a) e^{\lambda_{I}^{+}\left(\log a^{+}\right)} \Xi(a) J(a) d a \\
& \left.\leq \sqrt{d(\delta)} \int_{\overline{A^{+}}}|f|_{0}(a) e^{\left|\lambda_{I}^{+}\right| \sigma(a)} \Xi(a) J(a) d a \text { (since }\left|\log a^{+}\right|=\sigma(a)\right) \\
& \leq \sqrt{d(\delta)} \int_{\overline{A^{+}}}|f|_{0}(a) e^{\left|\lambda_{I}\right| \sigma(a)} \Xi(a) J(a) d a\left(\text { since }\left|\lambda_{I}^{+}\right|=\left|\lambda_{I}\right|\right) .
\end{aligned}
$$

Taking (3.3) into account, we see that the above integral is bounded by a constant depending only on $f$ whenever $\left|\lambda_{I}\right|<r$. Since $\lambda \mapsto\left(\Phi_{\bar{\lambda}, \delta}^{*}(x)\right)_{i j}$ is holomorphic in $\lambda$ for each fixed $x$, it follows by applying Morera's theorem in conjunction with Fubini's theorem that $\widehat{f^{\delta}}(\lambda)_{i j}$ is holomorphic in the domain $\left\{\lambda \in \mathfrak{a}_{\mathbb{C}}^{*}:\left|\lambda_{I}\right|<r^{\prime}\right\}$ for any $r^{\prime}, 0<r^{\prime}<r$. In particular the restriction of $\widehat{f^{\delta}}(\lambda)_{i j}$ to $\mathfrak{a}^{*}$ is real analytic, which contradicts the assumption that $S_{f}$ has finite measure. This proves our proposition.

Now we will prove Theorem 3.1.

Proof. By the above proposition $f \in L^{1}(X) \cap L^{2}(X)$.

Using the right $K$-invariance of $f$ and $\sigma$, we will show that the given condition implies

$$
I=\int_{G} \int_{\mathfrak{a}^{*}}|f(x)|\|\widehat{f}(\lambda)\|_{2} e^{\sigma(x)|\lambda|} e^{-\rho(H(x))} d x \mu(\lambda) d \lambda<\infty .
$$

On replacing $x$ by $x k^{-1}$ in the integral $I$, we get

$$
I=I(k)=\int_{G} \int_{\mathfrak{a}^{*}}|f(x)|\|\widehat{f}(\lambda)\|_{2} e^{\sigma(x)|\lambda|} e^{-\rho\left(H\left(x k^{-1}\right)\right)} d x \mu(\lambda) d \lambda .
$$

Therefore

$$
I=\int_{K} I(k) d k=\int_{K} \int_{G} \int_{\mathfrak{a}^{*}}|f(x)|\|\widehat{f}(\lambda)\|_{2} e^{\sigma(x)|\lambda|} e^{-\rho\left(H\left(x k^{-1}\right)\right)} d x \mu(\lambda) d \lambda d k .
$$

Since $\Xi(x)=\Xi\left(x^{-1}\right)=\int_{K} e^{-\rho\left(H\left(x^{-1} k\right)\right)} d k$ (see [7], p. 159), we conclude from Fubini's theorem and (3.1) that $I<\infty$.

Using the decomposition $G=K A N$ and the left $K$-invariance of $\sigma$, we get

$$
\int_{K \times A \times N} \int_{\mathfrak{a}^{*}}|f(k a n)|\|\widehat{f}(\lambda)\|_{2} e^{\sigma(a n)|\lambda|} e^{-\rho(\log a)} e^{2 \rho(\log a)} d k d a d n \mu(\lambda) d \lambda<\infty .
$$

Since $\sigma(a n) \geq \sigma(a)$ for all $a \in A$ and $n \in N$ ([7], Lemma 6.2.7 ii), we have from the above that

$$
\int_{\mathfrak{a}^{*}} \int_{K \times A} \mathcal{R}|f|(k, a)\|\widehat{f}(\lambda)\|_{2} e^{\sigma(a)|\lambda|} d k d a \mu(\lambda) d \lambda<\infty .
$$

We fix a $\delta \in \widehat{K}_{M}$. Now as $|\mathcal{R} f(k, a)| \leq \mathcal{R}|f|(k, a)$ and $|\lambda(\log a)| \leq|\lambda| \sigma(a)$, using (2.4), we have

$$
\int_{\mathfrak{a}^{*}} \int_{A}\left\|\mathcal{A} f^{\delta}(a)\right\|_{2}\|\widehat{f}(\lambda)\|_{2} e^{|\lambda(\log a)|} d a \mu(\lambda) d \lambda<\infty .
$$


Since $\left\|\widehat{f^{\delta}}(\lambda)\right\|_{2} \leq\|\widehat{f}(\lambda)\|_{2}$, we arrive at

$$
\int_{\mathfrak{a}^{*}} \int_{A}\left\|\mathcal{A} f^{\delta}(a)\right\|_{2}\left\|\widehat{f^{\delta}}(\lambda)\right\|_{2} e^{\left|\left\langle H_{\lambda}, \log a\right\rangle\right|} d a \mu(\lambda) d \lambda<\infty .
$$

In the above $\left\langle H_{\lambda}, \log a\right\rangle=B\left(H_{\lambda}, \log a\right)$ (see Section 2).

For $1 \leq i \leq d(\delta)$ and $1 \leq j \leq l$ we consider the $(i, j)$-th matrix entry of $\mathcal{A} f^{\delta}(a)$ and of $\widehat{f^{\delta}}(\lambda)$. Then the above implies that

$$
\int_{\mathfrak{a}^{*}} \int_{A}\left|\mathcal{A} f^{\delta}(a)_{i j}\right|\left|\widehat{f^{\delta}}(\lambda)_{i j}\right| e^{\left|\left\langle H_{\lambda}, \log a\right\rangle\right|} d a \mu(\lambda) d \lambda<\infty .
$$

Recall that as $f \in L^{1}(X), f^{\delta} \in L^{1}(X)$ and hence the matrix entries $\mathcal{A} f_{i j}^{\delta} \in$ $L^{1}(A)$ (see the discussion following Definition 2.3). By Lemma 2.4, $\left(\mathcal{A} f_{i j}^{\delta}, \widehat{f}^{\delta}{ }_{i j}\right)$ is a Euclidean Fourier transform pair. Using the identification of $\mathfrak{a}^{*}$ and of $A$ with $\mathbb{R}^{n}$, we can write the above inequality as

$$
\int_{\mathbb{R}^{n}} \int_{\mathbb{R}^{n}}|F(a) \| \widetilde{F}(\lambda)| e^{|\langle\lambda, a\rangle|} d a \mu(\lambda) d \lambda<\infty,
$$

where $\langle$,$\rangle is the Killing inner product of \lambda$ and $a$. Here $F(a)=\mathcal{A} f^{\delta}(a)_{i j}$ and hence $F \in L^{1}\left(\mathbb{R}^{n}\right)$ and $\widetilde{F}(\lambda)=\widehat{f^{\delta}}(\lambda)_{i j}$. We recall (see the proof of Proposition 3.2) that $\widehat{f^{\delta}}(\lambda)_{i j}$ is a real analytic function on $\mathfrak{a}^{*}$.

For $n \geq 2$ (for $n=1$ see Remark 3.3 after the proof), using polar coordinates $\lambda=s \omega$, we have from (3.4) for almost every $\omega \in S^{n-1}$ that

$$
\int_{\mathbb{R}^{n}} \int_{\mathbb{R}^{\prime}}|F(a)||\widetilde{F}(s \omega)| e^{|\langle s \omega, a\rangle|}|s|^{n-1} \mu(s \omega) d a d s<\infty .
$$

For any such $\omega \in S^{n-1}$, let $g_{\omega}(r)=\mathcal{R}^{E} F(\omega, r)$, for $r \in \mathbb{R}$. Then $g_{\omega} \in L^{1}(\mathbb{R})$ and $\widetilde{g}_{\omega}(s)=\widetilde{F}(s \omega)=\widetilde{F}(\lambda)$ (see Section 2). Now,

$$
\begin{aligned}
& \int_{\mathbb{R}} \int_{\mathbb{R}}\left|g_{\omega}(r)\right|\left|\widetilde{g}_{\omega}(s)\right| e^{|r s|}|s|^{n-1} \mu(s \omega) d r d s \\
\leq & \int_{\mathbb{R}} \int_{\mathbb{R}} \int_{\langle a, \omega\rangle=r}|F(a)| d \sigma_{a}|\widetilde{F}(s \omega)| e^{|r s|}|s|^{n-1} \mu(s \omega) d r d s \\
= & \int_{\mathbb{R}^{n}} \int_{\mathbb{R}}|F(a)||\widetilde{F}(s \omega)| e^{|\langle a, s \omega\rangle|}|s|^{n-1} \mu(s \omega) d a d s .
\end{aligned}
$$

In the above we have used that $\bigcup_{r \in \mathbb{R}}\{a \mid\langle a, \omega\rangle=r\}=\mathbb{R}^{n}$ and for $a \in \mathbb{R}^{n}$ which satisfies $\langle a, \omega\rangle=r,|r s|=|\langle a, s \omega\rangle|$. Hence, using (3.5), we get for almost every $\omega \in S^{n-1}$ that

$$
\int_{\mathbb{R}} \int_{\mathbb{R}}\left|g_{\omega}(r)\right|\left|\widetilde{g}_{\omega}(s)\right| e^{|r s|}|s|^{n-1} \mu(s \omega) d r d s<\infty .
$$

We rewrite (3.6) as

$$
\int_{\mathbb{R}}\left|\widetilde{g}_{\omega}(s)\right||s|^{n-1} \mu(s \omega) M_{1}(s) d s<\infty,
$$

where $M_{1}(s)=\int_{\mathbb{R}}\left|g_{\omega}(r)\right| e^{|r s|} d r$.

From (3.7) we have $\left|\widetilde{g}_{\omega}(s)\right||s|^{n-1} \mu(s \omega) M_{1}(s)<\infty$ for almost every $s \in \mathbb{R}$. Now $s \mapsto \mu(s \omega)$ and $s \mapsto \widetilde{g}_{\omega}(s)$ are both real analytic for all fixed $\omega \in S^{n-1}$. Hence the zero set of $\mu(s \omega)\left|g_{\omega}(s)\right|$ is at most countable. Furthermore $|s|^{n-1}>0$ for $s \in$ $\mathbb{R} \backslash\{0\}$. This implies that $M_{1}(s)<\infty$ for almost every $s \in \mathbb{R}$, that is, $\sup \{|s| \mid s \in$ $\left.\mathbb{R}, M_{1}(s)<\infty\right\}=\infty$. Note that $M_{1}(s)$ is even in $s$ and an increasing function in $|s|$. 
This implies that $M_{1}(s)<\infty$ for all $s \in \mathbb{R}$ and it is bounded on any compact subset and therefore it is locally integrable. Hence we have $\int_{-M}^{M}\left|\widetilde{g}_{\omega}(s)\right||s|^{n-1} M_{1}(s) d s<\infty$ for any $M>0$, that is

$$
\int_{\mathbb{R}} \int_{-M}^{M}\left|g_{\omega}(r)\right|\left|\widetilde{g}_{\omega}(s)\right| e^{|r s|}|s|^{n-1} d r d s<\infty .
$$

In the asymptotic estimate (2.6) of $\mu(\lambda)$ we use polar coordinates for $\lambda=s \omega$ to see that $|\langle\lambda, \alpha\rangle|=|s||\langle\omega, \alpha\rangle|$ and for almost every $\omega \in S^{n-1},\langle\omega, \alpha\rangle \neq 0$ for all $\alpha \in \Sigma_{0}^{+}$. Thus for any such fixed $\omega$ and for $|\lambda|=|s|>M$ for some large $M>0$, $\mu(\lambda)$ can be substituted by a rational function of $|s|$ say, $P_{1}(|s|) / P_{2}(|s|)$ where $P_{1}$, $P_{2}$ are polynomials and $\operatorname{deg} P_{1}>\operatorname{deg} P_{2}$. Clearly we can choose the above $M$ suitably such that for $|s|>M,|s|^{n-1} P_{1}(|s|)>P_{2}(|s|)$.

Hence we obtain from $(3.7)$

$$
\int_{\mathbb{R}} \int_{|s|>M}\left|g_{\omega}(r)\right|\left|\widetilde{g}_{\omega}(s)\right| e^{|r s|}|s|^{n-1} \frac{P_{1}(|s|)}{P_{2}(|s|)} d r d s<\infty
$$

which implies that

$$
\int_{\mathbb{R}} \int_{|s|>M}\left|g_{\omega}(r)\right|\left|\widetilde{g}_{\omega}(s)\right| e^{|r s|} d r d s<\infty .
$$

Inequalities (3.8) and (3.9) together imply

$$
\int_{\mathbb{R}} \int_{\mathbb{R}}\left|g_{\omega}(r)\right|\left|\widetilde{g}_{\omega}(s)\right| e^{|r s|} d r d s<\infty .
$$

Now we can apply Theorem 1.1 to conclude that $\widetilde{g}_{\omega}(s)=0$ for almost every $s \in \mathbb{R}$. As this is true for almost every $\omega \in S^{n-1}$, we get $\widehat{f}^{\delta}{ }_{i j}(\lambda)=0$ for every $\lambda \in \mathfrak{a}^{*}$ since $\widehat{f}_{i j}(\lambda)$ is continuous. The Plancherel theorem now implies that $f^{\delta}=0$ almost everywhere. Since $\delta \in \widehat{K}_{M}$ is arbitrary, we conclude that $f(x)=0$ for almost every $x \in X$.

Remark 3.3. When rank of $X$ is 1 , the inequality (3.4) becomes

$$
\int_{\mathbb{R}} \int_{\mathbb{R}}|F(a) \| \widetilde{F}(\lambda)| e^{|\langle\lambda, a\rangle|} d a \mu(\lambda) d \lambda<\infty .
$$

We can eliminate the Plancherel density $\mu(\lambda)$ in the above inequality basically because $\mu(\lambda) \longrightarrow \infty$ as $|\lambda| \longrightarrow \infty$ (see [13] for details). Now the Euclidean Beurling theorem implies that $\widetilde{F}(\lambda)=\widehat{f^{\delta}}(\lambda)=0$ for all $\lambda \in \mathfrak{a}^{*}$.

\section{SECOND VERSION}

In this section we use the Helgason Fourier transform to formulate the following Beurling theorem.

Theorem 4.1. Let $f \in L^{1}(X) \cap L^{2}(X)$. If

$$
\int_{K \times \mathfrak{a}^{*}} \int_{X}|f(x)||\tilde{f}(\lambda, k)| e^{|\lambda(A(x, k M))|} e^{-\rho(A(x, k M))} d x d k \mu(\lambda) d \lambda<\infty,
$$

then $f=0$ almost everywhere. 
Notice that the Helgason Fourier transform is an integral transform whose kernel is $e_{\lambda, k}(x)=e^{(-i \lambda+\rho) A(x, k M)}$. These functions $e_{\lambda, k}$ are eigenfunctions of the LaplaceBeltrami operator of the Riemannian symmetric space $X$ which are constant on horocycles. Thus they are the analogues of the plane waves in Euclidean space. The relation between the weight $e^{|\langle\lambda, x\rangle|}$ in Beurling's theorem on $\mathbb{R}$ and the kernel $e^{-i \lambda x}$ of the Fourier transform on $\mathbb{R}$ motivates us to formulate the above theorem for symmetric spaces.

Proof. We can change the integration over $X$ in (4.1) to integration over $G$; that is, we consider $f$ as a right $K$-invariant function in $L^{1}(G) \cap L^{2}(G)$ and start with the condition

$$
\int_{K \times \mathfrak{a}^{*}} \int_{G}|f(x)||\widetilde{f}(\lambda, k)| e^{|\lambda(A(x, k M))|} e^{-\rho(A(x, k M))} d x d k \mu(\lambda) d \lambda<\infty .
$$

This is possible as the integrand in the $G$-integral above is right $K$-invariant. Writing $A(x, k M)=-H\left(x^{-1} k\right)$, substituting $x=k y$, and using the left $G$-invariance of the measure $d x$, we get from (4.2) that

$$
I=\int_{K \times \mathfrak{a}^{*}} \int_{G}|f(k y) \| \widetilde{f}(\lambda, k)| e^{\left|\lambda\left(H\left(y^{-1}\right)\right)\right|} e^{-\rho\left(H\left(y^{-1}\right)\right.} d y d k \mu(\lambda) d \lambda<\infty .
$$

We use the Iwasawa decomposition $G=A N K$ and write $y=a n k_{1}$ according to this decomposition to get

$$
\begin{aligned}
I=\int_{K \times \mathfrak{a}^{*}} \int_{A \times N \times K} \mid & f\left(\operatorname{kank}_{1}\right)|| \widetilde{f}(\lambda, k) \mid \\
& \times e^{\left|\lambda\left(H\left(k_{1}^{-1} n^{-1} a^{-1}\right)\right)\right|} e^{-\rho\left(H\left(k_{1}^{-1} n^{-1} a^{-1}\right)\right)} d a d n d k_{1} d k \mu(\lambda) d \lambda .
\end{aligned}
$$

Using the right $K$-invariance of $f$ and the left $K$-invariance of $H$,

$$
I=\int_{K \times \mathfrak{a}^{*}} \int_{A \times N}|f(k a n)||\widetilde{f}(\lambda, k)| e^{\left|\lambda\left(H\left(n^{-1} a^{-1}\right)\right)\right|} e^{-\rho\left(H\left(n^{-1} a^{-1}\right)\right)} d a d n d k \mu(\lambda) d \lambda .
$$

Now since $A$ normalizes $N$ and $H$ is right $N$-invariant, we have $H\left(n^{-1} a^{-1}\right)=$ $H\left(a^{-1}\right)=-\log a$. Hence

$$
\begin{aligned}
I & =\int_{K \times \mathfrak{a}^{*}} \int_{A} e^{\rho(\log a)} \int_{N}|f(k a n)| d n|\tilde{f}(\lambda, k)| e^{|\lambda(\log a)|} d a d k \mu(\lambda) d \lambda \\
& =\int_{K} \int_{\mathfrak{a}^{*}} \int_{A} \mathcal{R}|f|(k, a)|\widetilde{f}(\lambda, k)| e^{|\lambda(\log a)|} d a d k \mu(\lambda) d \lambda .
\end{aligned}
$$

Thus for almost every $k \in K$,

$$
\int_{\mathfrak{a}^{*}} \int_{A} \mathcal{R}|f|(k, a)|\widetilde{f}(\lambda, k)| e^{|\lambda(\log a)|} d a \mu(\lambda) d \lambda<\infty .
$$

Since $|\mathcal{R} f(k, a)| \leq \mathcal{R}|f|(k, a)$, we finally arrive at

$$
\int_{\mathfrak{a}^{*}} \int_{A}|\mathcal{R} f(k, a)||\widetilde{f}(\lambda, k)| e^{|\lambda(\log a)|} d a \mu(\lambda) d \lambda<\infty .
$$

Recall that for almost every $k \in K$ for which the above inequality is valid, $\mathcal{R} f(k, a)$ is an $L^{1}$-function on $A$ and $(\mathcal{R} f(k, a), \tilde{f}(\lambda, k))$ is a Euclidean Fourier transform pair ([10], p. 276). Using the identification of $A$ and of $\mathfrak{a}^{*}$ with $\mathbb{R}^{n}$, we can write the above inequality as

$$
\int_{R^{n}} \int_{\mathbb{R}^{n}}|F(a)||\widetilde{F}(\lambda)| e^{|\lambda(a)|} d a \mu(\lambda) d \lambda<\infty,
$$


where $F \in L^{1}\left(\mathbb{R}^{n}\right)$. That is, we obtain the inequality (3.4) of the previous section. Using the argument there, we conclude that $\widetilde{F}(\lambda)=0$ for every $\lambda \in \mathbb{R}^{n}$ (since $\widetilde{F}$ is continuous), i.e., for almost every $k \in K, \widetilde{f}(\lambda, k)=0$. The Plancherel theorem for $X$ now shows that $f=0$ almost everywhere.

Corollary 4.2. Let $f \in L^{1}(X) \cap L^{2}(X)$. If

$$
\int_{K \times \mathfrak{a}^{*}} \int_{X}|f(x)||\widetilde{f}(\lambda, k)| e^{(|\lambda|+|\rho|)|(A(x, k M))|} d x d k \mu(\lambda) d \lambda<\infty,
$$

then $f=0$ almost everywhere.

As

$$
|\lambda||A(x, k M)| \geq|\lambda(A(x, k M))|
$$

and

$$
|\rho||A(x, k M)| \geq|\rho(A(x, k M))| \geq-\rho(A(x, k M)),
$$

the corollary follows from the above theorem.

\section{Consequences}

As mentioned in the introduction, the theorems of Hardy, Morgan, Cowling and Price, Gelfand and Shilov, etc., all follow from our Beurling's theorem. The mutual dependencies of these theorems can be schematically displayed as follows:

$$
\begin{array}{clc}
\text { Beurling } \Rightarrow \quad \text { Gelfand and Shilov } & \Rightarrow & \text { Cowling and Price } \\
\Downarrow & & \Downarrow \\
\text { Morgan } & \Rightarrow & \text { Hardy }
\end{array}
$$

This shows that Beurling's theorem is the master theorem. Some of the latter theorems (which follow from Beurling's) were proved independently on symmetric spaces in recent years by many authors (see $[15,3,12,14,16,6]$, etc.). The statements of those theorems and proofs of the above implications in the case of rank 1 symmetric spaces can be found in [13]. The proofs in the case of higher rank are similar. For the sake of completeness we show how the theorem of Gelfand and Shilov follows from Beurling's theorem.

Theorem 5.1 (Gelfand and Shilov). Let $f \in L^{2}(X)$. Suppose $f$ satisfies

$$
\begin{aligned}
& \text { (1) } \int_{X}|f(x)| e^{\frac{(\alpha \sigma(x))^{p}}{p}} \Xi(x) d x<\infty, \\
& \text { (2) } \int_{\mathfrak{a}^{*}}\|\widehat{f}(\lambda)\|_{2} e^{\frac{(\beta|\lambda|)^{q}}{q}} \mu(\lambda) d \lambda,
\end{aligned}
$$

where $1<p<\infty, \frac{1}{p}+\frac{1}{q}=1, \alpha, \beta$ are positive constants. Then if $\alpha \beta \geq 1$, we have $f=0$ almost everywhere.

Proof. Since $\frac{\alpha^{p}}{p} \sigma(x)^{p}+\frac{\beta^{q}}{q}|\lambda|^{q} \geq \alpha \beta \sigma(x)|\lambda|$, the assertion follows immediately from Theorem 3.1. 


\section{REFERENCES}

[1] Anker J. -P. A basic inequality for scattering theory on Riemannian symmetric spaces of the noncompact type, Amer. Jr. Math. 113 (1991), no. 3, 391-398. MR1109344 (92k:43008) MR1109344 (92k:43008)

[2] Bonami, A; Demange, B.; Jaming, P. Hermite functions and uncertainty principles for the Fourier and the windowed Fourier transforms, Rev. Mat. Iberoamericana 19 (2003), no. 1, 23-55. MR1993414 (2004f:42015) MR1993414 (2004f:42015)

[3] Cowling, M.; Sitaram, A.; Sundari, M. Hardy's uncertainty principle on semisimple groups. Pacific J. Math. 192 (2000), no. 2, 293-296. MR1744570 (2001c:22007) MR1744570 (2001c:22007)

[4] Eguchi, M. Some properties of Fourier transform on Riemannian symmetric spaces. Lectures on harmonic analysis on Lie groups and related topics, pp. 9-43, Lectures in Math., 14, Kinokuniya Book Store, Tokyo, 1982. MR0683464 (84h:43026) MR683464 (84h:43026)

[5] Folland, G. B. Introduction to partial differential equations. Princeton University Press, Princeton, NJ, 1995. MR1357411 (96h:35001) MR1357411 (96h:35001)

[6] Folland, G. B.; Sitaram, A. The uncertainty principle: a mathematical survey. J. Fourier Anal. Appl. 3 (1997), no. 3, 207-238. MR1448337 (98f:42006) MR1448337 (98f:42006)

[7] Gangolli, R., Varadarajan, V. S. Harmonic analysis of spherical functions on real reductive groups. Ergebnisse der Mathematik und ihrer Grenzgebiete, 101. Springer-Verlag, Berlin, 1988. MR0954385 (89m:22015) MR954385 (89m:22015)

[8] Havin, V., Jöricke, B. The uncertainty principle in harmonic analysis. Ergebnisse der Mathematik und ihrer Grenzgebiete (3), 28. Springer-Verlag, Berlin, 1994. MR1303780 (96c:42001) MR1303780 (96c:42001)

[9] Helgason, S. The Abel, Fourier and Radon Transforms on Symmetric Spaces. Indagationes Mathematicae 16 (2005), 531-551. MR2313637

[10] Helgason S. Geometric Analysis on Symmetric Spaces, Mathematical Surveys and Monographs, 39. American Mathematical Society, Providence, RI, 1994 MR 96h:43009 MR1280714 (96h:43009)

[11] Hörmander, L. A uniqueness theorem of Beurling for Fourier transform pairs. Ark. Mat. 29 (1991), no. 2, 237-240. MR1150375 (93b:42016) MR1150375 (93b:42016)

[12] Narayanan, E. K.; Ray, S. K. L ${ }^{p}$ version of Hardy's theorem on semisimple Lie groups. Proc. Amer. Math. Soc. 130 (2002), no. 6, 1859-1866 MR1887035 (2003a:22009) MR1887035 (2003a:22009)

[13] Sarkar, R. P.; Sengupta, J. Beurling's Theorem for Riemannian Symmetric Spaces of noncompact type preprint 2005.

[14] Sengupta, J. The uncertainty principle on Riemannian symmetric spaces of the noncompact type. Proc. Amer. Math. Soc. 130 (2002), no. 4, 1009-1017 MR1873774 (2003a:43009)

[15] Sitaram, A.; Sundari, M. An analogue of Hardy's theorem for very rapidly decreasing functions on semi-simple Lie groups. Pacific J. Math. 177 (1997), no. 1, 187-200. MR1873774 (2003a:43009) MR1444779 (99a:22018)

[16] Thangavelu, S. An introduction to the uncertainty principle. Hardy's theorem on Lie groups. Progress in Mathematics, 217. Birkhäuser Boston, Inc., Boston, MA, 2004. MR2008480 (2004j:43007) MR2008480 (2004j:43007)

Stat-Math Unit, Indian Statistical Institute, 203 B. T. Rd., Calcutta 700108, India E-mail address: rudra@isical.ac.in

School of Mathematics, Tata Institute of Fundamental Research, Homi Bhabha Rd., Mumbai 400005, India

E-mail address: sengupta@math.tifr.res.in 